\title{
Asymmetric Special Theory of Relativity
}

\author{
Nils H. Abramson \\ Industrial Metrology, Royal Institute of Technology, Stockholm, Sweden \\ Email: nilsa@iip.kth.se
}

How to cite this paper: Abramson, N.H. (2018) Asymmetric Special Theory of Relativity. Journal of Modern Physics, 9, 471-478. https://doi.org/10.4236/jmp.2018.93033

Received: December 14, 2017 Accepted: February 21, 2018

Published: February 24, 2018

Copyright $\odot 2018$ by author and Scientific Research Publishing Inc. This work is licensed under the Creative Commons Attribution International License (CC BY 4.0).

http://creativecommons.org/licenses/by/4.0/

\begin{abstract}
Einstein's Special Theory of Relativity (SR) relates time dilation to the velocity between the observer and the observed object as if they are identical. Our new theory breaks this symmetry by relating the velocity of the object not directly to the observer, but instead to the center of gravity of object and observer. The reason why such a mass influence has not been reported might be that the mass of the observer in most experiments is much greater than that of the object, for example when earth is observing, satellites or detectors are studying nuclear masses.
\end{abstract}

\section{Keywords}

Asymmetry, Special Relativity, Time Dilation, Observers' Influence, Mass Influence, Satellite, Holodiagram

\section{Introduction}

Einstein's Special Relativity (SR) treats how velocity slows a travelling clock by time dilation. The higher the velocity, the more time dilation. Let us study an empty universe with two objects, $A$ and $B$, close together and fixed to each other. Let us assume that the only way to produce a velocity between $A$ and $B$ is by releasing a pushing massless spring between them. The result will be that the center of gravity $C$ stays fixed while $A$ and $B$ will start to separate at velocities depending on their masses. Thus, the momentums of $A$ and $B$ stay constant while velocity and energy are highest on the lightest object, resulting in greatest time dilation.

However, in SR there is strangely enough not mentioned any influence of the masses of observer and object. This situation has caused a lot of questions [1] [2]. We will try to find the answer to these problems by referring velocity to the center of gravity of the two studied objects instead of their direct relative velocities. In the following chapter, we will start by studying how this definition 
of velocity also influences different dynamic situations.

\section{Calculation of Balance on Weighing Scales}

$$
\left\{\begin{array}{l}
A C+C B=L \\
A C \cdot M=C B \cdot m
\end{array}\right.
$$

The resulting tilting momentum at $C$, as seen in Figure 1, is

$$
p=M \frac{L m}{M+m}-m \frac{L M}{M+m}=0 .
$$

The unknown mass of $B(m)$ is found by variation of $C B$. The result of the weighing procedure is

$$
m=M(A C / C B) .
$$

\subsection{Centrifugal Force at $C$ Is Zero}

If there was a rotation of $\theta$ around $C$ the horizontal centrifugal force at $A$ and $B$ would be $\theta^{2} \cdot m(A C)$ respective $\theta^{2} \cdot M(B C)$ resulting in that $C$ will stay fixed in balance because the two centrifugal forces are equal (Figure 2).

\subsection{Gravitational Attraction Force $F$ at $C$}

$$
F=M g / R^{2}
$$

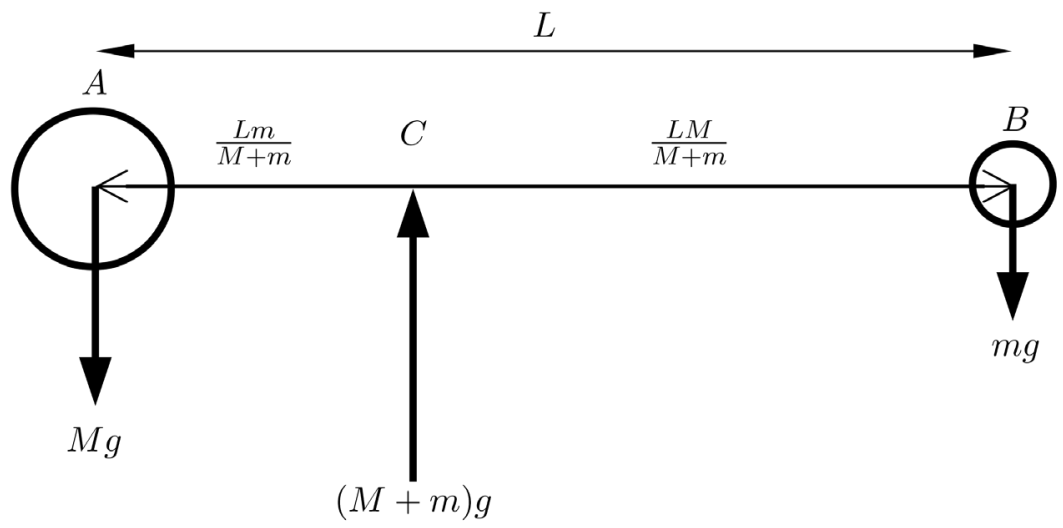

Figure 1. Ancient method of measuring the mass $m$ of $B$. The point of balance is found where the momentum caused by gravity results in no tilting force at $C$.

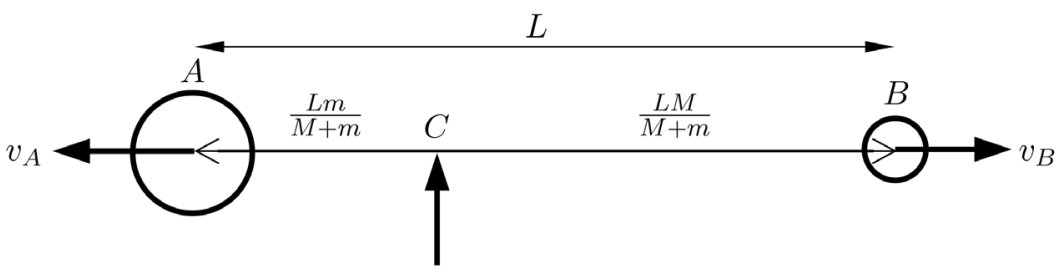

Figure 2. Substituting the vertical arrows of gravity of Figure 1 by horizontal arrows of velocity, we find that the velocity at $C$ must be zero to conserve the momentum of gravity. 
The force between $A$ and $C$ is

$$
F=\frac{M(M+m) g}{(m / M+m)^{2}} .
$$

The force between $B$ and $C$ is

$$
F=\frac{m(M+m) g}{(M / M+m)^{2}} .
$$

Thus, there is a gravitational balance at $C$, which is also proved by astronomy.

\section{Velocity and Time Dilation in Einstein's Special Theory of Relativity [3]}

However, there is strangely enough not mentioned in SR any influence of the masses of observer and object. This situation has caused a lot of questions [1]-[8]. We will find the answer to these problems by referring velocity to the center of gravity of the two studied objects instead of their direct relative velocities.

First let us study the equation of Einstein's time dilation:

$$
t_{v}=\frac{t_{0}}{\sqrt{1-\frac{v^{2}}{c^{2}}}}=\gamma t_{0},
$$

where $t_{0}$ is the length of a tick of a clock that is stationary $(v=0)$ and $t_{v}$ the length of a tick of a clock that is moving at the velocity $v . c$ is the constant velocity of light. $\gamma$ the conventional accepted symbol of Equation (1), and $v$ the velocity of the clock in relation to the observer.

The velocity of a single object in a totally empty universe cannot be explained. Before Einstein the velocity $v$ was defined as velocity in relation to a universe. In SR $v$ was defined as the relative velocity between observer and observed object. If, however, there exists two objects $A$ and $B$, their velocity $v$ in relation to each other can be defined and used as an important factor in e.g. Einstein's definition of time dilation.

We propose that $v$ is defined as the velocity of the observer in relation to the center of gravity of observer and observed object. As example of the importance of this definition of velocity we study how the length of a click of a travelling clock depends on its mass.

Let us study an empty universe with two objects $A$ and $B$ close together and fixed to each other. Let us assume that the only way to produce a velocity between $A$ and $B$ is by releasing a pushing massless spring between them. The result will be that the centre of gravity $C$ stays fixed while $A$ and $B$ will start to separate at velocities depending on their masses. Thus, the momentums of $A$ and $B$ will stay constant while velocity and energy will be highest on the lightest object, resulting in greatest time dilation. Here I want to promote this situation into a postulate:

Independent of how the two objects are accelerated, the relativistic velocity 
$v$ should always be related to their common center of inertia $C$ for the masses $M$ and $m$.

\section{Time Dilation on a Satellite [4] [5]}

The most accurate measurements of relativistic time dilation have been made by comparing the results of clocks on a satellite $t_{v}$ respective on the stationary earth $t_{0}$. Let us first again study Equation (3) of the time dilation introduced by Einstein in SR.

$$
t_{v}=\frac{t_{0}}{\sqrt{1-\frac{v^{2}}{c^{2}}}}=\gamma t_{0}
$$

A clock with the length of one tick a second at velocity zero is placed at both $M$ and $m$. Where $t_{v}$ is the time displayed by the travelling clock $B, t_{0}$ that of the stationary clock $A$ and $v$ is the velocity of $B$ in relation to $A$. In the following we will not state which one is stationary but only study the time of one object in relation to the other. In the case of the measurement of the satellite in relation to the earth, we understand there must be a factor missing in Equation (3) that breaks the symmetry of the two objects. The most obvious factor to include into the equation is their difference in mass.

Let us make a calculation where $M$ is the mass of earth, while $m$ is the mass of a satellite and assume that their common point of gravity is stationary. The result will be that $v$ of Equation (3) will be different when the time dilation is measured from the earth or from the satellite. Our calculations are still based on the limits of SR. Thus, no influence of acceleration or gravity (as in general relativity [6]) is included in our results where $v_{M}$ is the velocity of earth and $v_{m}$ that of the satellite. Our new proposal is that $v$ is not the velocity of the satellite in relation to earth, but the velocity of the satellite in relation to the common point of gravity, $C$, of satellite and earth.

$$
\begin{gathered}
M v_{A}=m v_{B} \\
v_{A}+v_{B}=v \\
v_{C}=0
\end{gathered}
$$

Combining Equations (5) and (6) results in:

$$
\left\{\begin{array}{l}
v_{A}=v \frac{m}{M+m} \\
v_{B}=v \frac{M}{M+m}
\end{array} .\right.
$$

Thus the time dilation for $B$ as measured from $C$ is

$$
t_{B}=\frac{t_{0}}{\sqrt{1-\left(\frac{M}{M+m} \cdot \frac{v}{c}\right)^{2}}},
$$

and the time dilation for $A\left(t_{A}\right)$ as measured from $C$ is 


$$
t_{A}=\frac{t_{0}}{\sqrt{1-\left(\frac{m}{M+m} \cdot \frac{v}{c}\right)^{2}}},
$$

and the time dilation of $B\left(t_{B}\right)$ as measured from $A$ :

$$
\frac{t_{B}}{t_{A}}=\frac{t_{0} \sqrt{1-\left(\frac{m}{M+m} \cdot \frac{v}{c}\right)^{2}}}{t_{0} \sqrt{1-\left(\frac{M}{M+m} \cdot \frac{v}{c}\right)^{2}}} .
$$

Equation (10) represents the most accurate result of the time dilation as calculated from the idea of asymmetric distribution of the velocity combined to SR. However, Equation (10) looks rather complicated and to get a more simplified view we study a more graphic visualization method, e.g. the Holodiagram.

\section{Holodiagram [7]}

The ordinary symmetric holodiagram (Figure 3 ) visualizes in a simple way to compute the time dilation caused by a constant velocity $v$ of the point source of light $A$ and a point source of observation $B$ in relation to a stationary object point $C$. The two points $A$ and $B$ are together on a train that runs at a constant velocity $v$, During the time light travels $A C B$ the train travels the distance $A O B$. In order to meet at the same time at the same place $B$, both must travel during the same time $t_{v}$. Thus, using the Pythagorean theorem we get

$$
\left\{\begin{array}{l}
\left(c t_{v}\right)^{2}=\left(c t_{0}\right)^{2}+\left(v t_{v}\right)^{2} \\
t_{v}=\frac{t_{0}}{\sqrt{1-\frac{v^{2}}{c^{2}}}}=\gamma t_{0}=\frac{t_{0}}{\sqrt{1-\sin ^{2} \alpha}}=\frac{t_{0}}{\cos \alpha}
\end{array}\right.
$$

In asymmetric special relativity, the velocity at $A\left(v_{A}\right)$ is different from that of $B\left(v_{B}\right)$. Thus, we compare the time of one click at the clock of $B$ (sat) compared to that of $A$ (earth).

\section{Our Solution to the Twin-Paradox [8]}

If the two identical twins have identical masses their velocities in relation to their common centre of gravity will be identical and thus there will be no difference in velocity and thus the time measured by the clocks will be identical. This result is independent of the velocity direction of each twin (thus no paradox). However, also as measured from earth there will be identical time dilations of the two twins. This time dilation will be identical to the time dilation introduced by Einstein in his special theory of relativity (Equation (3)), because the mass of earth is much greater than that of the twins.

Graphic derivation of the satellite time dilation, where $t_{B} / t_{A}$ of Equation (8) is represented by $(F-m) /(F-M) . t_{A} / t_{0}$ of Equation (7) shows the increase of time for light passing from $F$ to $m$ (Figure 4 ). 


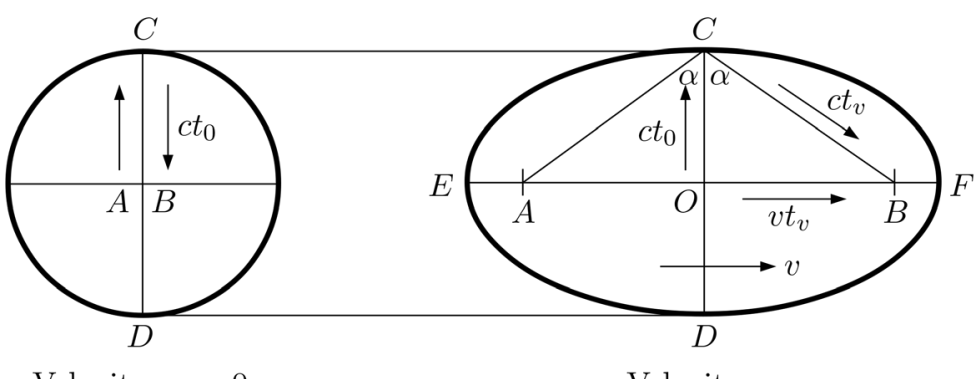

Velocity: $v=0$

Velocity: $v$

Figure 3. $A$ is a point source of an ultrashort light pulse, while $B$ is a point of ultrashort observation made later. If the separation of $A$ and $B$ is zero, only points on a sphere are observed. If the separation is larger, the sphere is elongated to an ellipsoid. In holography, this separation is usually static, but in relativity it is dynamic, caused by velocity $v$.

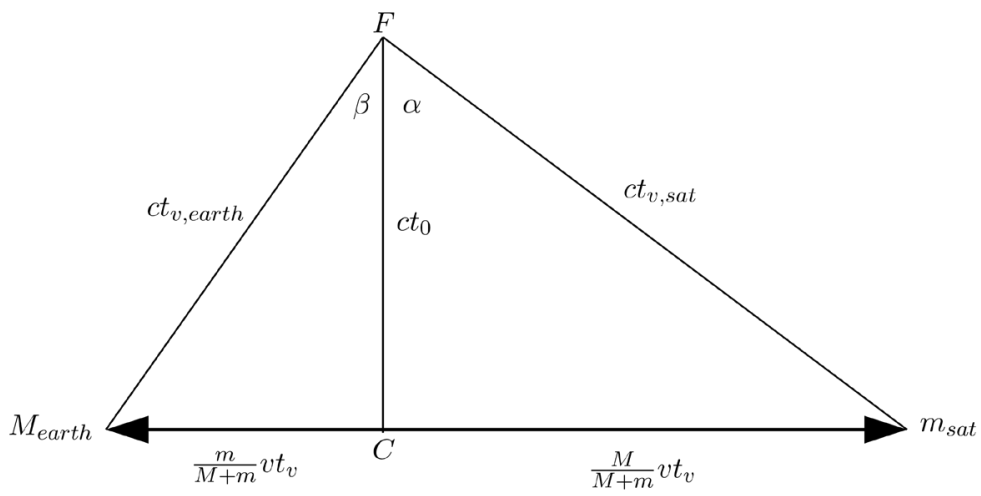

Figure 4. Asymmetric derivation of $t_{v, \text { sat }} / t_{v, \text { earth }}$.

$$
t_{v, \text { sat }} / t_{v, \text { earth }}=\cos \beta / \cos \alpha
$$

$m \gg M$ results in $t_{v} / t_{0}=$ inverted value of time dilation.

\section{Relativistic Approximation}

Let us go back to Equation (10) which represents the accurate result of the time dilation as calculated from the idea of asymmetric distribution of the velocity combined to special relativity. However, the equation looks rather complicated and to get a more simplified view we want to find a short approximation. Let us start by studying the approximation of relativistic kinetic energy $e$ into classic energy when velocity is much lower than $c$.

$$
e=\frac{m c^{2}}{\sqrt{1-\frac{v^{2}}{c^{2}}}}-m c^{2} \approx \frac{m c^{2}}{1-\frac{v^{2}}{2 c^{2}}}-m c^{2} \approx m c^{2}\left(1+\frac{v^{2}}{2 c^{2}}\right)-m c^{2}=\frac{m v^{2}}{2}
$$

$e$ represents the kinetic energy which is the total energy minus the nuclear energy $\left(m c^{2}\right)$. Substituting $m c^{2}$ by $t_{0}$ we get the following equation:

$$
t_{v}=\frac{t_{0}}{\sqrt{1-\frac{v^{2}}{c^{2}}}}-t_{0} \approx \frac{t_{0}}{1-\frac{v^{2}}{2 c^{2}}}-t_{0} \approx t_{0}\left(1+\frac{v^{2}}{2 c^{2}}\right)-t_{0}=t_{0}+\frac{t_{0} v^{2}}{2 c^{2}}-t_{0}=\frac{t_{0} v^{2}}{2 c^{2}}
$$




$$
\begin{aligned}
& \left\{\begin{array}{l}
v_{A}=\frac{m l}{m+M} \text { thus } A C=\frac{m l}{m+M} \\
v_{B}=\frac{M}{m+M} \text { thus } C B=\frac{M l}{m+M}
\end{array}\right. \\
& \left\{\begin{array}{l}
t_{A}=\frac{1}{\sqrt{1-\left(\frac{v_{B}}{c}\right)^{2}}} \\
t_{B}=\frac{1}{\sqrt{1-\left(\frac{v_{A}}{c}\right)^{2}}}
\end{array}\right. \\
& \frac{t_{B}}{t_{A}} \approx \frac{\sqrt{1-\left(\frac{v_{A}}{c}\right)^{2}}}{\sqrt{1-\left(\frac{v_{B}}{c}\right)^{2}}} \approx \sqrt{1-\left(\frac{v_{A}}{c}\right)^{2} \cdot \sqrt{1+\left(\frac{v_{B}}{c}\right)^{2}}}=1+\frac{1}{c^{2}}\left(v_{B}^{2}-v_{A}^{2}\right)
\end{aligned}
$$

thus

$$
\frac{t_{B}}{t_{A}} \approx 1+\frac{v^{2}\left(M^{2}-m^{2}\right)}{c^{2}\left(M^{2}+m^{2}\right)} .
$$

When the mass of the satellite is very small compared to the mass of earth the accepted equation of time dilation in SR is correct. Clocks on satellite appear slower.

When the mass of the satellite were equal to the mass of earth there will be no time dilation of the satellite as measured from earth. This result is in disagreement with SR. There will be no difference on the clocks.

When the mass of the satellite were larger than that of earth there were observed a time increase. Clocks on the satellite will be faster as studied from earth. This result is in disagreement with SR.

Equation (15) shows separation of clicks $t_{B}$ of the clocks on $B$ as compared with those $t_{A}$ on $A$. We study what happens if the mass of the satellite is increased and find that when the mass $m$ of $B$ is increased its time dilation $t_{B} / t_{A}$ is decreased (Figure 5).

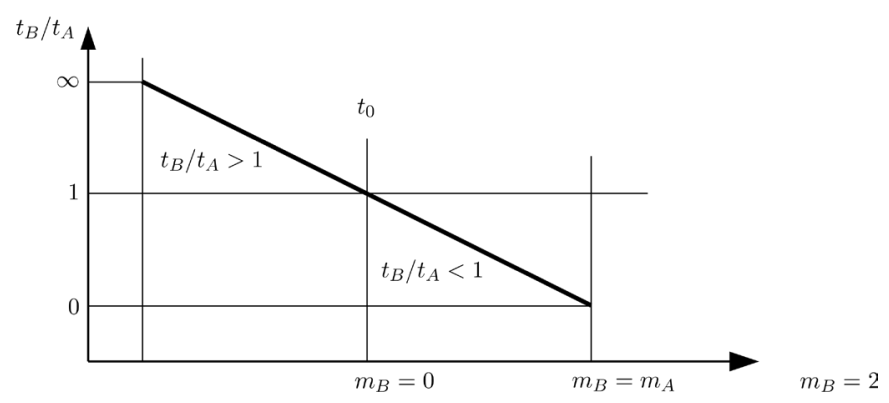

Figure 5. The mass (earth) of the observer at $A\left(m_{A}\right)$ is constant, while we study $t_{B} / t_{A}$ when we let the mass (satellite) at $B\left(m_{B}\right)$ increase from zero to $2 m_{A}$. 


\section{Comparing Einstein's Special Relativity to Our New Asymmetric Relativity}

The reason that there has been measured a time dilation of clocks in the GPS satellites compared to clocks on earth is simply that the mass of earth is much bigger than that of the satellite. No such conclusion is given in SR.

When the time dilation is observed (measured) from the heaviest body, the result agrees with SR. However, if it is made from the lightest body, the result will instead be time acceleration. Thus the satellite observes clocks on earth to run faster. No such conclusion is given in SR.

Solution to the twin paradox is that the heaviest twin will see his lighter twin to age slower. If the masses of the twins are identical, they will age with the same time and thus see no time dilation.

A rocket with the length of 100 meters flying at relativistic velocity can be enclosed in a tunnel of the length of 50 meters if the rocket is much lighter than earth.

When the speed of light is measured locally it will always be $c$. However, when measured from a fast moving satellite, the speed of light at earth will appear faster than $c$.

\section{Acknowledgements}

Finally I want to thank the Royal Institute of Technology for the necessary contacts and especially Jonny Gustafsson for interesting discussions and for valuable and practical advice.

\section{References}

[1] Burniston, B.G. (1967) Bulletin of the Institute of Physics and the Physical Society, 18, 71-77.

[2] Kunst, E. (2014) Natural Science, 6, 226-247. https://doi.org/10.4236/ns.2014.64027

[3] Einstein, A. (1905) Annals of Physics, 322, 891-921. https://doi.org/10.1002/andp.19053221004

[4] Ashby, N. (2002) Physics Today, 55, 41-47. https://doi.org/10.1063/1.1485583

[5] van Flandern, T. (2003) Apeiron, 10, 69-86.

[6] Einstein, A. (1916) Annals of Physics, 354, 769-822. https://doi.org/10.1002/andp.19163540702

[7] Abramson, N. (2014) Applied Optics, 53, 2398-2404. https://doi.org/10.1364/AO.53.002398

[8] Sato, M. (2005) Twin Paradox Experiment of the Transverse Doppler Shift. arXiv:physics/0502007v2. 